\title{
Magnetic Field Decay, or Just Period-Dependent Beaming?
}

\author{
Joeri van Leeuwen \& Frank Verbunt \\ Astronomical Institute, Utrecht University, PO Box 80000, 3508 TA \\ Utrecht, The Netherlands
}

\begin{abstract}
Several recent papers conclude that radio-pulsar magnetic fields decay on a time-scale of $10 \mathrm{Myr}$, apparently contradicting earlier results. We have implemented the methods of these papers in our code and show that this preference for rapid field decay is caused by the assumption that the beaming fraction does not depend on the period. When we do include this dependence, we find that the observed pulsar properties are reproduced best when the modeled field does not decay. When we assume that magnetic fields of new-born neutron stars are from a distribution sufficiently wide to explain magnetars, the magnetic field and period distributions we predict for radio are pulsars wider than observed. Finally we find that the observed velocities overestimate the intrinsic velocity distribution.
\end{abstract}

\section{Introduction}

Neutron star magnetic fields are determined in both X-ray and radio pulsars. The cyclotron resonance scattering features in the X-ray spectrum of accreting neutron stars allow an estimate of the field strengths. The results lie in a narrow range between $(1-4) \times 10^{12} \mathrm{G}$ (Makishima et al. 1999), for both young systems and systems as old as $10^{8}$ years. This strongly suggests that neutron star magnetic fields do not decay spontaneously.

In contrast, three arguments have been put forward for rapid ( $\sim 10 \mathrm{Myr})$ field decay in radio pulsars. Firstly, the anti-correlation of characteristic age $\left(\tau_{c} \propto P \dot{P}^{-1}\right)$ and magnetic field strength, or more correctly, torque $\left(\propto[P \dot{P}]^{1 / 2}\right)$, seems to indicate magnetic field decay. It is better explained by the strong dependence of the quantities plotted.

Second, field decay could explain the scarcity of far-away pulsars, as they stop shining before they cover large distances. Yet since these distances are derived from dispersion measures, they will be systematically underestimated for pulsars beyond the Galactic gas layer (Bhattacharya \& Verbunt 1991), causing an apparent lack of far-away pulsars.

Third, if magnetic fields or torques do not decay, one expects more pulsars with long periods than are observed. A decrease in visibility with time could also explain this shortage; in fact, both Vivekanand \& Narayan (1981) and Lyne \& Manchester (1988) find that pulsars with longer periods have significantly narrower beams, making it less likely for them to be detected. 


\section{The Population Synthesis Code}

In a pulsar population synthesis code, one models the birth, life and death of radio pulsars and compares each simulated sample with the real sample. Certain pulsars (nearby, slow, bright ones for example) are more easily discovered than others and will be overrepresented in the sample. To account for these selection effects, one must explicitly model the surveys in which pulsars are detected.

Our code is a parallelized version of the one described by Bhattacharya et al. (1992) and Hartman et al. (1997) and runs on TERAS, the 1024-processor $1 \mathrm{Tflop} \mathrm{s}^{-1}$ supercomputer at SARA. In short, the algorithm is as follows: in the initial settings we assume distributions for the pulsar properties at birth, and fix the time-scale for exponential magnetic field decay. The properties of each simulated pulsar are drawn from the initial distributions. We evolve the magnetic field and period, calculate the orbit though the Galaxy and determine the size of the radio beam. We then check whether any of the incorporated surveys discover the pulsar, and repeat the loop until we have detected 2000 simulated pulsars. We compare their properties to those of real pulsars, vary the input settings and restart the synthesis, until the optimum solution is reached.

\section{Magnetic Field Decay}

Bhattacharya et al. (1992) and Hartman et al. (1997) found that models with long magnetic field decay times reproduced the observations best. Forcing the use of a short decay time, the simulation would only resemble the observed data after the introduction of a second pulsar population, with longer periods at birth, as was also found by Narayan \& Ostriker (1990).

Cordes \& Chernoff (1998) investigate the velocities and spin-down laws in 49 young pulsars. They assume a constant beaming fraction and find a magnetic field decay time of less than $10 \mathrm{Myr}$. Arzoumanian et al. (2002) use a perioddependent beaming model to generate pulse profiles. They do not, however, use this new beaming model to fit for the magnetic field decay time, but mention the value found by Cordes \& Chernoff (1998) instead: less than $10 \mathrm{Myr}$. We have implemented pulse profiles in the way described by Arzoumanian et al. (2002), but still find that the observed pulsar properties are best reproduced in a model without field decay. However, if we follow Cordes \& Chernoff (1998) in assuming that the beaming fraction does not depend on the period, we reproduce their result and find that the best model has a decay time of order $10 \mathrm{Myr}$.

Arzoumanian et al. (2002) further argue that all pulsars with the same $P$ and $\dot{P}$ have the same intrinsic (angle-averaged) luminosity and that the observed luminosity depends on the observation angle and interstellar scintillation. As the width of the resulting observed luminosity distribution is of the same magnitude as the intrinsic spread in the Narayan \& Ostriker (1990) luminosity function, the two descriptions are computationally almost identical.

Gonthier et al. (2002) simulate Galactic populations of radio and gammaray pulsars. With a beaming fraction constant with period, their best model has magnetic field decay in $5 \mathrm{Myr}$.

Although the exact relation is still subject of debate, it is clear that the beaming fraction decreases with the pulse period (Vivekanand \& Narayan 1981; 

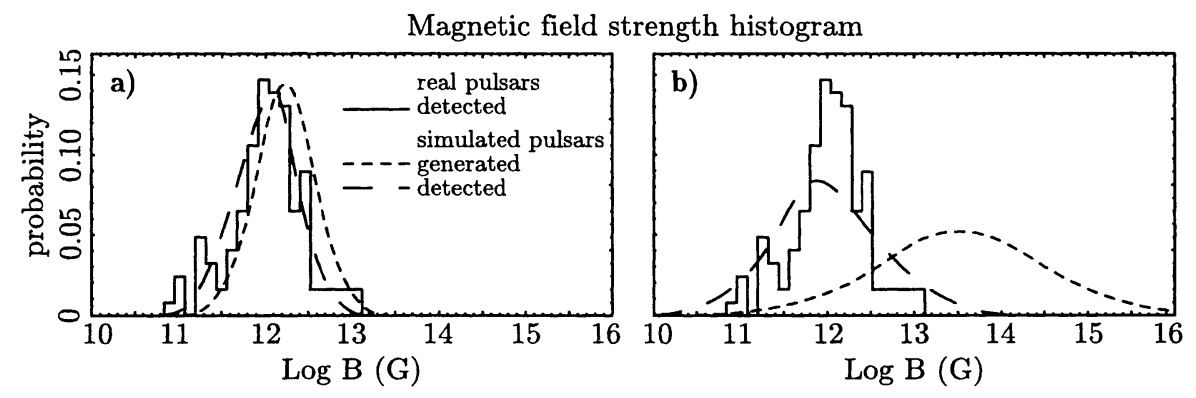

Figure 1. Single magnetic field distributions for the best model for: (a) radio pulsars (Kolmogorov-Smirnov probability $p=0.56$ ); (b) radio pulsars and magnetars $(p=0.03)$

Lyne \& Manchester 1988). If we force our code to disregard this relation, as Cordes \& Chernoff (1998), Gonthier et al. (2002) and Arzoumanian et al. (2002) chose to do, we also need magnetic-field decay on a time-scale of around $10^{7} \mathrm{yr}$ to reproduce the observed period distributions.

When we do include period dependence of the beaming fraction and search for the best solution, the no-decay case is much more probable than decay on a 10 Myr time scale (van Leeuwen \& Verbunt 2004).

\section{Magnetars}

Most of the pulsars we detect in our code were born near the Sun, which makes us mostly sensitive to the local birth rate. In our best model (no magnetic field decay) we need a local birth rate of $2 \mathrm{kpc}^{-2} \mathrm{Myr}^{-1}$, which can be produced by OB-association stars with masses over $10 \mathrm{M}_{\odot}$ (Hartman et al. 1997). In this model, the magnetic field strength is drawn from a log-normal distribution with center and width $10^{12.3 \pm 0.3} \mathrm{G}$. This makes no neutron stars with magnetic fields higher than $10^{14} \mathrm{G}$. To make a substantial number of these, as well as normal radio pulsars, we can shift and widen the distribution to $10^{13.5 \pm 0.9} \mathrm{G}$. This increases the underlying neutron star birth rate by roughly a factor of four. However, as the input magnetic field distribution is wider, the simulated period distribution and magnetic field distribution are both unacceptably wider than is observed (see Fig. 1). It therefore seems implausible that radio pulsars and magnetars are formed from a single neutron star magnetic field distribution.

\section{Velocities}

In previous work on pulsar speeds (Helfand \& Tademaru 1977; Lyne \& Lorimer 1994; Cordes \& Chernoff 1998) one argument keeps returning: fast pulsars quickly move away from the plane, and so from our telescopes, either to escape our Galaxy or to spend much time far away from it while they reverse direction. Many of these far-away pulsars escape detection, biasing the observed sample to the slow and sedentary pulsars (see Fig. 2[a]). 

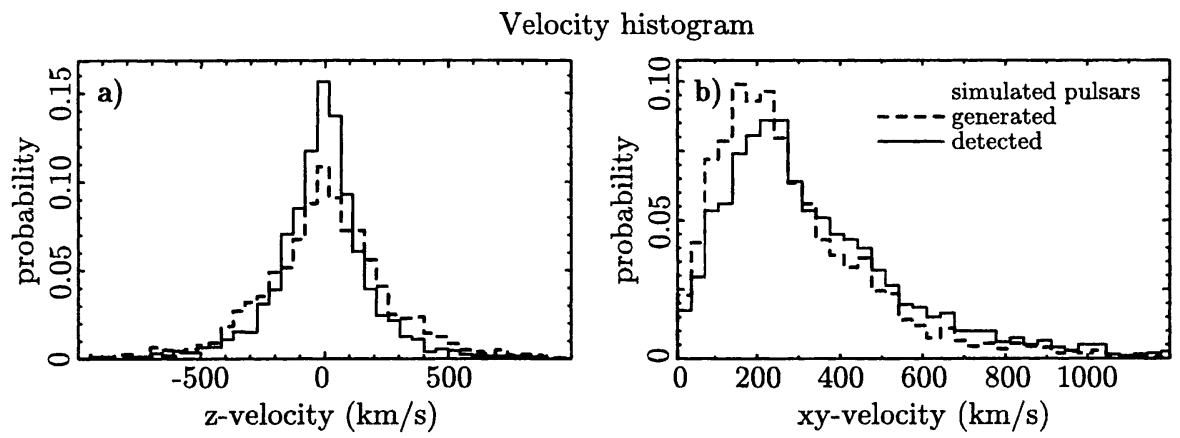

Figure 2. Velocity distribution in directions (a) perpendicular and (b) parallel to the Galactic plane, for the generated and detected populations of simulated radio pulsars.

This argument is valid in the $z$-direction only; in a three-dimensional world, the situation is different (see also Hansen \& Phinney 1997). Seen from the Earth, most pulsars are born toward the Galactic Center. Of these, the high-speed ones can travel the largest distances and these diffuse from the center outward, towards our part of the Galaxy (Fig. 2[b]). We find the effect of radial drift to be stronger than the observational selection effect in the $z$-direction: on the whole, our simulated detected pulsars move faster than the parent population (van Leeuwen \& Verbunt 2004).

\section{References}

Arzoumanian, Z., Chernoff, D. F., \& Cordes, J. M. 2002, ApJ, 568, 289

Bhattacharya, D., \& Verbunt, F. 1991, A\&A, 242, 128

Bhattacharya, D., Wijers, R. A. M. J., Hartman, J. W., \& Verbunt, F. 1992, A\&A, 254, 198

Cordes, J. M., \& Chernoff, D. F. 1998, ApJ, 505, 315

Gonthier, P. L., Ouellette, M. S., Berrier, J., O'Brien, S., \& Harding, A. K. 2002, ApJ, 565, 482

Hansen, B., \& Phinney, E. S. 1997, MNRAS, 291, 569

Hartman, J. W., Bhattacharya, D., Wijers, R., \& Verbunt, F. 1997, A\&A, 322, 477

Helfand, D. J., \& Tademaru, E. 1977, ApJ, 216, 842

Lyne, A. G., \& Lorimer, D. R. 1994, Nature, 369, 127

Lyne, A. G., \& Manchester, R. N. 1988, MNRAS, 234, 477

Makishima, K., Mihara, T., Nagase, F., \& Tanaka, Y. 1999, ApJ, 525, 978

Narayan, R., \& Ostriker, J. P. 1990, ApJ, 352, 222

van Leeuwen, J., \& Verbunt, F. 2004, A\&A, in preparation

Vivekanand, M., \& Narayan, R. 1981, J. Astrophys. Astr., 2, 315 